\title{
Tipificación estructural y rentabilidad de explotaciones ovinas de carne
}

\author{
Louiza Chekmam ${ }^{1, *}$, María Teresa Maza-Rubio ${ }^{1}$ y Luis Pardos²
}

1 Departamento de Ciencias Agrarias y del Medio Natural, Facultad de veterinaria. Universidad de Zaragoza. Miguel Servet 177, 50013 Zaragoza, España

2 Departamento de Ciencias Agrarias y del Medio Natural, Escuela Politécnica Superior de Huesca, Universidad de Zaragoza. Carretera Cuarte s/n, 22071 Huesca, España

\section{Resumen}

El sector ovino de carne está experimentando un fuerte retroceso en España y en la Unión Europea. Se trata de un tipo de ganadería que desempeña un importante papel en zonas desfavorecidas por su contribución a la sostenibilidad económica, social y medioambiental, siendo importante garantizar su viabilidad económica. El objetivo genérico del presente trabajo ha sido analizar las posibles relaciones entre los resultados económicos de una muestra de explotaciones de ovino de carne ubicadas en Aragón (España) y determinados indicadores estructurales. Previamente se realizó una tipificación para estudiar su variabilidad. La muestra la componen 126 ganaderías aragonesas de ovino de carne. Se utilizan las medias de las variables durante un período que oscila entre 5 y 24 años dentro del periodo 1993-2016. Se realizó un análisis factorial sobre diez variables estructurales y se identificaron cuatro factores a partir de los cuáles se obtuvieron dos grupos significativamente diferentes en cuanto a sus características estructurales. Se eligió la renta disponible por unidad de trabajo como indicador del resultado económico de las explotaciones, y se calculó teniendo en cuenta o no la retribución de la mano de obra familiar. Mediante una regresión lineal múltiple se estableció su dependencia con las variables estructurales previamente analizadas. Se puede concluir que la optimización del número de ovejas por unidad de mano de obra y por hectárea de la superficie agrícola útil, son aspectos a tener en cuenta a la hora de mejorar la rentabilidad y la viabilidad de las explotaciones ovinas de carne.

Palabras clave: Ovino de carne, estructura explotación, resultados económicos.

\section{Structrural tipyfication and profitability of sheep meat farms}

\section{Abstract}

The sheep meat sector is experiencing a strong recoil in Spain and in the European Union. It is a type of livestock that plays an important role in disadvantaged areas for its contribution to economic, social and environmental sustainability, being important to ensure its economic viability. The generic objective of this work has been to analyze the possible relationship between the economic results of a sample of sheep meat farms located in Aragon (Spain) and certain structural indicators. Previously a typification was done to study their variability. The sample is made up of 126 Aragonese meat sheep

\footnotetext{
* Autor para correspondencia: kahina.lahna79@hotmail.fr
}

Cita del artículo: Chekmam L, Maza MT, Pardos L (2021). Tipificación estructural y rentabilidad de explotaciones ovinas de carne. ITEA-Información Técnica Económica Agraria 117(2): 191-207. https://doi.org/10.12706/itea.2020.023 
farms. The variables' means are used during a period that varies between 5 and 24 years within the 19932016 period. A factor analysis was performed on ten structural variables and four factors were identified from which two significantly different groups of farms were obtained in terms of their structural characteristics. The gross margin per total labor unit was chosen as an indicator of the economic result of farms, and was calculated considering or not the remuneration of family labor. By means of a multiple linear regression the dependence of this economic result on structural variables previously analyzed was established. It can be concluded that the optimization of the number of ewes per total labor unit and per hectare of the used agricultural area, are aspects to be considered when improving the profitability and viability of sheep meat farms.

Keywords: Meat sheep, farm structure, economic results.

\section{Introducción}

A pesar del reconocimiento que la Política Agraria Común (PAC) otorga al sector ovino por su contribución a la sostenibilidad social, económica y medioambiental (MAGRAMA, 2016), el censo de ganado ovino ha experimentado un claro retroceso en los últimos años tanto en España como en los países de nuestro entorno. En el periodo 2008-2018 el número de cabezas de ganado ovino disminuyó un 26,2 \% en España (MAPA, 2019), y un 7,5 \% en el conjunto de la Unión Europea (EUROSTAT, 2019). El descenso fue más acusado hasta 2013, observándose después una estabilización del censo.

Distintos factores han contribuido a que el sector ovino de carne se encuentre en una situación crítica: el aumento constante de los costes de producción, el estancamiento de los precios de venta del producto, la tendencia a la baja en el consumo de carne de cordero y la concurrencia internacional de distintos países productores (Bernués y Olaizola, 2012; Marín-Bernal y Navarro-Ríos, 2014; Olaizola et al., 2014; Rodríguez-Ortega et al., 2018a), entre otros. En este contexto pueden adoptarse diferentes medidas que palíen al menos de forma parcial el paulatino declive del sector. Entre ellas pueden destacarse el fomento y la promoción del consumo de carne de ovino, el aumento de la rentabilidad de las explotaciones o la adopción de diferentes po- líticas que regulen los mercados y aumenten las ayudas a las explotaciones sobre todo aquellas relacionadas con los servicios ecosistémicos que prestan (Olaizola et al., 2008; Rodríguez-Ortega et al., 2018a,b).

De todas estas medidas el aumento de la rentabilidad ha sido citada por algunos autores (Benoit y Laignel, 2011; Olaizola et al., 2014) como una medida fundamental para disminuir la dependencia de las explotaciones de las actuales ayudas, cuya continuidad se ve amenazada en las sucesivas revisiones que va sufriendo la Política Agraria Común. Además de la rentabilidad, Pardos et al. (2008) han señalado que una mayor flexibilidad que permita la adaptación a los cambios de la política agraria afectará también positivamente a la supervivencia de las explotaciones ovinas de carne.

Sin embargo, la heterogeneidad existente en las explotaciones ovinas de carne motivada en parte por su dependencia del medio donde se ubican, con una disponibilidad de recursos muy variada, dificulta el que puedan diseñarse medidas que consigan el mismo efecto en el conjunto de las explotaciones. Una de las fuentes de heterogeneidad de las explotaciones son sus características estructurales determinadas por la disponibilidad y el uso de los diferentes factores de producción. El grado de aplicación de un factor en relación a otro permite calificar a las explotaciones como más o 
menos intensivas en su utilización. De forma que en los procesos de intensificación se combina una unidad del factor limitante con cantidades crecientes de otros factores, mientras que se habla de extensificación con relación al factor relativamente más abundante, cuando éste se combina con dosis decrecientes de los factores (Tirel, 1983).

La tipificación de las explotaciones ovinas se ha realizado con cierta frecuencia por distintos autores por su utilidad para comprender la diversidad del sector (Gaspar et al., 2008; López-i-Gelats et al., 2011; Gelasakis et al., 2012). No ha tenido tanto la finalidad de poner en marcha diferentes políticas que tengan como objetivo la ayuda y promoción de las explotaciones; aunque como ha señalado Valerio Cabrera et al. (2014), los estudios de tipificación y caracterización permiten realizar mejores planes y distribución de los recursos destinados a gestionar los factores determinantes que rodean a las explotaciones. Las medidas que apoyen a las explotaciones ovinas de carne son necesarias por cuanto, tanto en España como en otros países, la mayoría están localizadas en áreas desfavorecidas, de hecho en nuestro país el $80 \%$ del censo se ubica en áreas con dificultades específicas (Andersen et al., 2007; Ruiz-Mirazo et al., 2011; Toro-Mujica et al., 2015). Además se trata de un sector multifuncional cuyas explotaciones practican sistemas de explotación extensivos o semi-extensivos, con utilización de razas autóctonas adaptadas a las condiciones ambientales y con un importante papel en la prevención de incendios forestales, la fijación de población rural en áreas muy desfavorecidas para otras actividades, la protección y conservación de prados y la posibilidad de ofrecer productos diferenciados con valor añadido (Ruiz-Mirazo et al., 2011; Vázquez et al., 2014; Mena et al., 2014). La multifuncionalidad de los sistemas de ovino de carne les convierte en un elemento vital para el equilibrio medio ambiental en las zo- nas donde se localizan, existiendo una interacción mutua entre la viabilidad de los sistemas y la protección de la biodiversidad de los ecosistemas (Olaizola et al., 2014; Dubeuf et al., 2016; Bernués et al., 2018; Rodríguez-Ortega, 2018a,b).

El objetivo del presente trabajo es caracterizar un grupo de explotaciones ovinas en función de sus características estructurales e identificar diferentes tipos de explotación. Para ello se tendrán en cuenta los principales factores de producción y la combinación de los mismos que las define como más o menos intensivas en su uso. Finalmente se analizará su posible relación con los resultados económicos alcanzados. Un mejor conocimiento de los resultados económicos obtenidos por los distintos tipos de explotación puede ayudar a detectar cuáles son más vulnerables y por tanto tener un mayor riesgo de desaparición.

\section{Material y métodos}

Para la realización del presente trabajo se contó con una muestra de 126 explotaciones ovinas de carne localizadas en Aragón (España). Estas explotaciones se distribuyen a través de sus tres provincias: Zaragoza, Huesca y Teruel, y siguen un programa de gestión técnico-económica utilizado por una importante cooperativa ubicada en esta región (Oviaragón-Grupo Pastores). Dada la antigüedad del programa que comenzó en la década de los 90 y la continuidad de muchas de las explotaciones, los datos que utilizamos son en algunos casos las medias de los 24 años que van desde 1993 a 2016. Para cada explotación el dato medio de cada variable corresponde a la media de los años de los que se tienen registros. Lo prolongado del periodo estudiado hizo necesario que aquellas variables expresadas en unidades monetarias fueran convertidas a euros constantes del 2016. 
Se seleccionaron una serie de variables estructurales basándonos en estudios de explotaciones ovinas llevados a cabo anteriormente por distintos autores (Hamrouni 1993; Chertouh, 2005; Maza et al., 2008; Pardos et al., 2008; López-i-Gelats et al., 2011). Tras un análisis detallado de las variables más frecuentemente utilizadas, se seleccionaron 10 variables estructurales referidas a la superficie y su uso, a la mano de obra y al tamaño del rebaño (Tabla 1). Relacionadas con el factor tierra se incluyeron la superficie agrícola útil total (SAUT), el porcentaje que supone el regadío (\% Reg/SAUT), las hectáreas de arrendamientos forrajeros aprovechados a diente (AF), las hectáreas de cultivos forrajeros (HCF) y la relación de la superficie con la mano de obra (SAUT/UTAT). Asimismo, se incluyeron la variable número de ovejas (NO), y su relación con la superficie (NO/SAUT) y la mano de obra (NO/UTAT). Por último, se consideró el número de unidades de trabajo anual total (UTAT) y el porcentaje de trabajo familiar (\% UTF).
Para lograr el objetivo de la presente investigación se utilizaron análisis univariantes y multivariantes. El análisis univariante permitió realizar una descripción general de la muestra. Los análisis multivariantes fueron el análisis de componentes principales (ACP) para reducir el número de variables y facilitar el análisis posterior de los resultados, el análisis clúster bietápico para la obtención de grupos de explotaciones, y la regresión lineal múltiple para establecer relaciones de dependencia (Hair et al., 2014).

El análisis clúster permite la obtención de grupos de explotaciones lo más homogéneas posible dentro de cada grupo y lo más heterogéneas entre grupos. Se tuvieron en cuenta las puntuaciones factoriales de los individuos para realizar el análisis clúster. Con la variable de «pertenencia al clúster» se definieron las proporciones de los clústeres.

En el análisis de regresión lineal múltiple las variables predictoras fueron las diez variables estructurales utilizadas para realizar el análisis factorial, y como variable dependiente se

Tabla 1. Descripción de la muestra(1) por las variables estructurales.

Table 1. Description of the sample by structural variables.

\begin{tabular}{lcc}
\hline Datos estructurales & Media & $\begin{array}{c}\text { Deviación } \\
\text { estándar }\end{array}$ \\
\hline Número de Ovejas (NO) & 605,6 & 304 \\
Número de Unidades de Trabajo Anual Total (UTAT) & 1,32 & 0,49 \\
\% Unidades de Trabajo Familiar (\% UTF) & 90 & 18,0 \\
Número de Ovejas por Unidad de Trabajo Total (NO/UTAT) & 463,2 & 143,7 \\
Número de Ovejas por hectárea de Superficie Agrícola Útil Total (NO/SAUT) & 17,5 & 59 \\
Superficie Agrícola Útil por unidad de Trabajo Total (SAUT/UTAT) & 74,6 & 61,4 \\
Superficie Agrícola Útil Total (SAUT) & 95,7 & 87,8 \\
\% Regadío sobre Superficie Agrícola Útil Total (\% Reg/SAUT) & 22,2 & 32,3 \\
Arrendamiento forrajero (AF) & 461,3 & 791 \\
Hectáreas de cultivos forrajeros (HCF) & 21 & 22,7 \\
\hline
\end{tabular}

(1) Muestra total de 126 explotaciones ovinas de carne en Aragón. 
consideró la renta disponible por unidad de trabajo total (RDPUTAT). La renta disponible por unidad de trabajo puede considerarse como la medida más significativa de los resultados económicos obtenidos y un indicador de la posible continuidad de las explotaciones al incluir la productividad por oveja y el tamaño correcto del rebaño (Olaizola et al., 1996). En su cálculo puede incluirse o no la retribución de la mano de obra familiar dado el carácter eminentemente familiar de las explotaciones ovinas. En el presente trabajo se ha calculado de ambas formas.

En el análisis de regresión lineal múltiple, se utilizó el método por pasos para retener solo las variables independientes significativas en los modelos resultantes. El modelo genérico lineal se formuló de la siguiente manera:

$$
\begin{aligned}
& \text { RDPUTAT }=\beta_{0}+\beta_{1} X_{1}+\beta_{2} X_{2}+\beta_{3} X_{3}+ \\
& +\ldots \beta_{n} X_{n}+\mathrm{e}
\end{aligned}
$$

Donde RDPUTAT (la renta disponible por unidad de trabajo anual total) es la variable dependiente, $\beta_{0}$ es la constante de regresión, y $\beta_{1} \beta_{2} \beta_{3} \ldots \beta_{n}$ son coeficientes a estimar, $X_{1}, X_{2}$ $X_{3} \ldots X_{n}$ son las variables estructurales utilizadas y (e) es el error del modelo de la regresión. Los coeficientes de regresión se comprobaron usando la prueba t. El coeficiente de determinación $\left(R^{2}\right)$ se utilizó como criterio predictivo de éxito para el modelo de regresión (Drapper y Smith, 1998 citado por Sakar et al., 2011). Se ha utilizado el paquete SPSS versión 26 para ejecutar los análisis estadísticos.

La robustez de cada uno de los modelos fue validada de la siguiente manera: la ausencia de multicolinearidad se verificó utilizando el índice de tolerancia y el factor de inflación (FIV). Según Pérez (2005) un FIV grande y un índice de tolerancia pequeño pueden indicar posible presencia de colinealidad. Para verificar la correlación serial de los residuos se aplicó la prueba de Durbin Watson, que establece que un valor cercano a 2 indica que no existen problemas de autocorrelación.
Usualmente se considera que entre 1,5 y 2,5 debería existir independencia entre los residuos (Pineda Jaimes et al., 2011).

\section{Resultados y discusión}

\section{Descripción de la muestra}

El tamaño promedio de las 126 explotaciones estudiadas es de 605,6 ovejas, manejadas por 1,32 unidades de mano de obra total (tabla 1). Esta última cifra coincide con la obtenida por Chertouh (2005) con 1,32 UTAT, en explotaciones de ovino de carne en Aragón. Las unidades de mano de obra familiar representan aproximadamente el $90 \%$ del total de unidades de mano de obra, lo que significa que las explotaciones de la muestra son en su mayoría explotaciones familiares. Con respecto a la intensificación de la mano de obra, el número de ovejas por unidad del trabajo es el índice más utilizado. El número medio de ovejas por unidad del trabajo es de 463,2 ovejas/UTAT. En opinión de Pardos y Fantova (2007) los mejores resultados económicos se obtienen en explotaciones que manejan entre 400 y 600 ovejas por unidad de trabajo. La media de la superficie agrícola útil de estas explotaciones es de 95,7 ha, de las cuales el $22,2 \%$ son de regadío. Los cultivos forrajeros suponen 21 ha de media y los arrendamientos forrajeros para pastoreo a diente 461,3 ha. Como medidas de intensificación la carga ganadera (NO/SAUT) se sitúa en 17,5 ovejas/ha, al tiempo que se registra una media de 74,6 ha de superficie agrícola útil por unidad de mano de obra (SAUT/UTAT).

\section{Análisis de Componentes Principales}

El análisis factorial parte de la matriz de correlación entre las variables que analiza. La mayoría de los coeficientes de correlación entre las variables son significativos ( $p \leq 0,01$ o $p \leq 0,05$ ) (Complemento 1), por lo que puede realizarse el análisis (Sakar et al., 2011). 
Destaca la alta correlación positiva que presenta la variable número de ovejas (NO) con las variables número de unidades de trabajo total (UTAT), la intensificación de la mano de obra (NO/UTAT), las hectáreas de cultivos forrajeros (HCF), la superficie agrícola útil (SAUT) y el arrendamiento forrajero (AF). Por otro lado, el número de ovejas se correlaciona de forma significativa aunque con coeficiente negativo con el porcentaje de trabajo familiar (\% UTF). Estos tres principales factores productivos (el capital vivo, la mano de obra y la superficie) están correlacionados, y este resultado es similar al encontrado por Hamrouni (1993), quien afirmó que estos factores productivos manifestaban una relación de dimensión. Por otro lado, el número de unidades de trabajo total (UTAT) presenta correlación negativa con el porcentaje de mano de otra familiar (\% UTAF), y positiva con las variables de superficie SAUT, AF y HCF. Finalmente, la SAUT presenta una correlación positiva muy alta con SAUT/UTAT y negativa con el porcentaje de regadío.

Para reducir las dimensiones de los diez indicadores estructurales utilizados en esta sección, realizamos un análisis factorial que utilizó los componentes principales como método de extracción. Tanto el índice $\mathrm{KMO}=0,465$ como la prueba de esfericidad de Bartlett (Chi-cuadrado) fueron significativos $(p<0,000)$, lo que permitió seguir con la interpretación de los resultados. Las diez variables originales se sintetizaron en cuatro factores con valores propios superiores a 1 que explican el $71,78 \%$ de la varianza total, lo cual se considera un buen porcentaje (Hair et al., 2014). Realizamos el método Varimax para la rotación de factores; los resultados se muestran en la tabla 2.

El factor 1 explica el $28,45 \%$ de la varianza. A este factor le caracterizan dos de las diez variables iniciales; estas variables son el tamaño de la superficie agrícola útil (SAUT) y las hectáreas de la superficie agrícola útil por unidad de la mano de obra total (SAUT/UTAT).
El factor 2 explica $19,75 \%$ de la varianza. A este factor le caracterizan las unidades de la mano de obra total (UTAT) y el tamaño del rebaño (NO) y con signo negativo el porcentaje de unidades de trabajo familiar (\%UTF). Esta relación negativa entre el porcentaje de trabajo familiar y el tamaño del rebaño ha sido señalada por otros autores (Hamrouni, 1993; Chertouh, 2005).

El factor 3 explica el $13,42 \%$ de la varianza total. Al factor le caracterizan dos variables, hectáreas de cultivos forrajeros (HCF) y arrendamientos forrajeros (AF), por lo que una mayor superficie de cultivos forrajeros estaría asociada a un mayor arrendamiento de este tipo de aprovechamientos.

El factor 4 explica el 10,15\% de la varianza total. Le caracterizan las variables número de ovejas por unidad de mano de obra (NO/UTAT), la carga ganadera por hectárea de la SAUT (NO/SAUT) y el porcentaje del regadío sobre la SAUT (\% Reg/SAUT). Este factor caracteriza pues a explotaciones más intensivas en el uso de la tierra y del trabajo con más proporción de superficie regada.

\section{Análisis clúster}

Se han obtenido dos grupos de explotaciones. El primero incluye 90 explotaciones y el segundo 36. Los tamaños de los clústeres no están equilibrados, pero la calidad del clúster se considera suficiente (figura 1).

La realización del ANOVA $(p<0,0001)$ permite demostrar que cada grupo está relacionado de manera diferente con respecto a cada factor.

\section{Perfil de los grupos}

La tabla 3 muestra el perfil de los grupos. El grupo 1 está correlacionado negativamente con los cuatro factores y el grupo 2 esta correlacionado positivamente con los cuatro factores. 


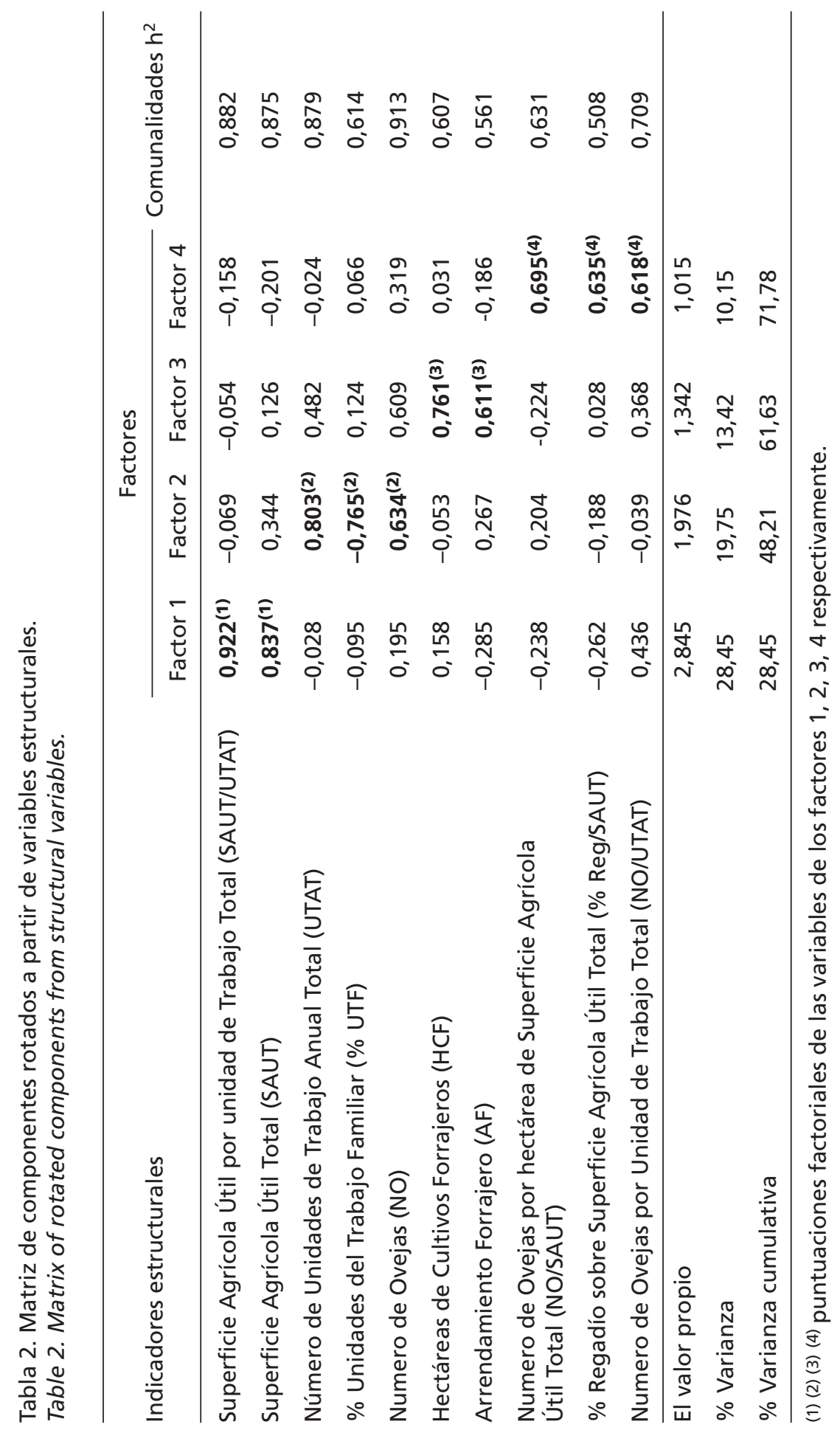




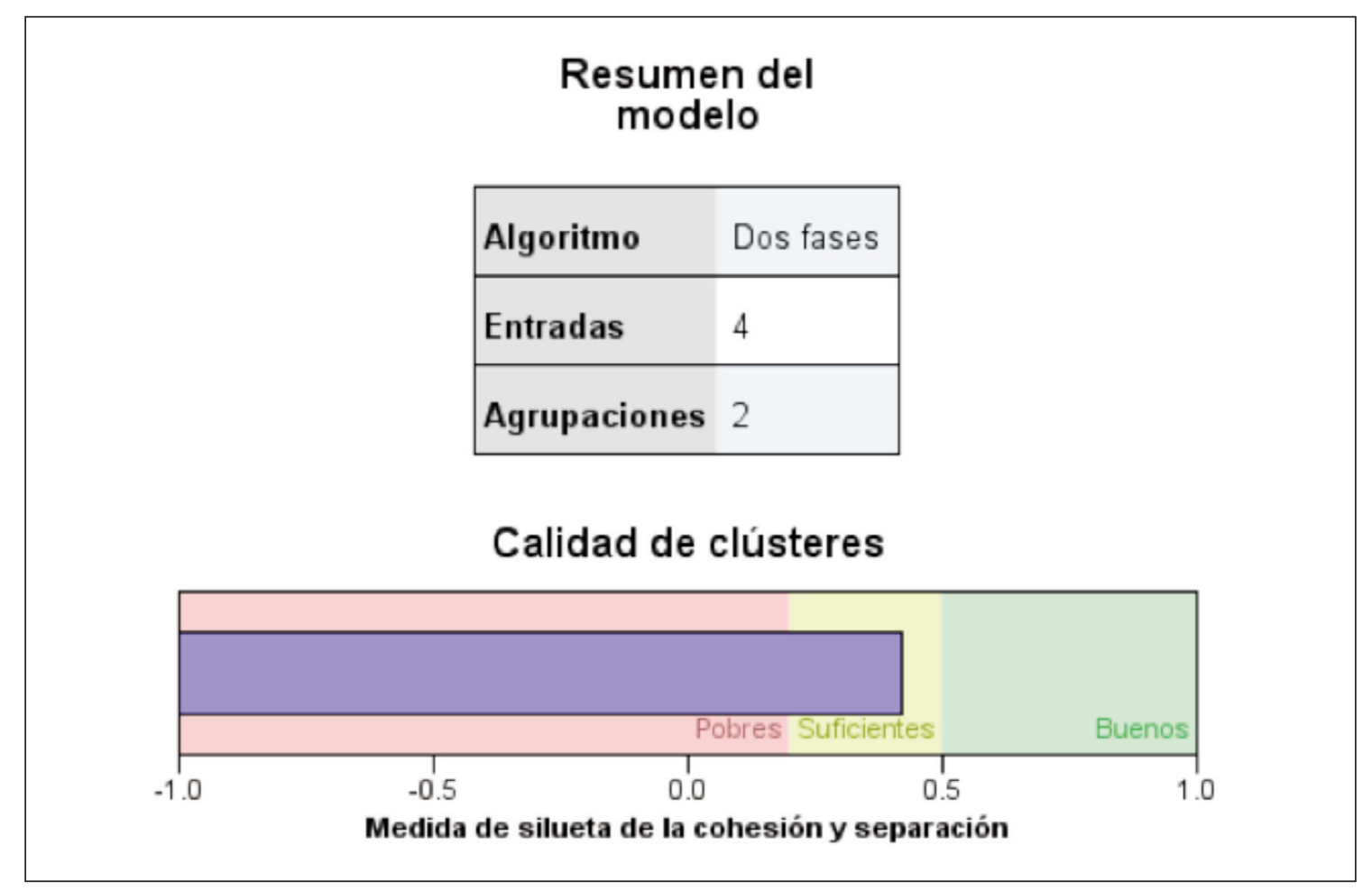

Figura 1. Calidad de los grupos.

Fuente: Output del análisis clúster.

Figure 1. Clusterts' quality.

Tabla 3. Perfiles de puntuaciones de los factores. Table 3. Profiles of the factors' scores.

\begin{tabular}{lll}
\hline Factores & Grupo 1 & Grupo 2 \\
\hline Factor 1 & $-0,1466566$ & 0,3666416 \\
Factor 2 & $-0,4538995$ & 1,1347487 \\
Factor 3 & $-0,1327536$ & 0,3318841 \\
Factor 4 & $-0,0706074$ & 0,1765185 \\
\hline
\end{tabular}

Para una descripción más detallada y una mejor caracterización de cada grupo, la variable "pertenencia a un grupo» se ha cruzado con las diez variables estructurales utilizadas en el presente trabajo. Los resultados se pre- sentan en la tabla 4. Se han añadido también las variables indicativas de sus resultados económicos, que actuarán como variables dependientes en el análisis posterior. Como puede observarse, la mayoría de las variables permiten diferenciar los dos grupos de explotaciones, a excepción de las variables NO/SAUT y \% Reg/SAUT. Los resultados económicos obtenidos por cada uno de los grupos no muestran diferencias significativas.

El grupo 1, que agrupa al 71,4 \% de las explotaciones, está formado por explotaciones pequeñas o medianas con respecto a la dimensión del rebaño, a la superficie agrícola útil total, y a las unidades de la mano de obra total. Tiene más importancia la mano 


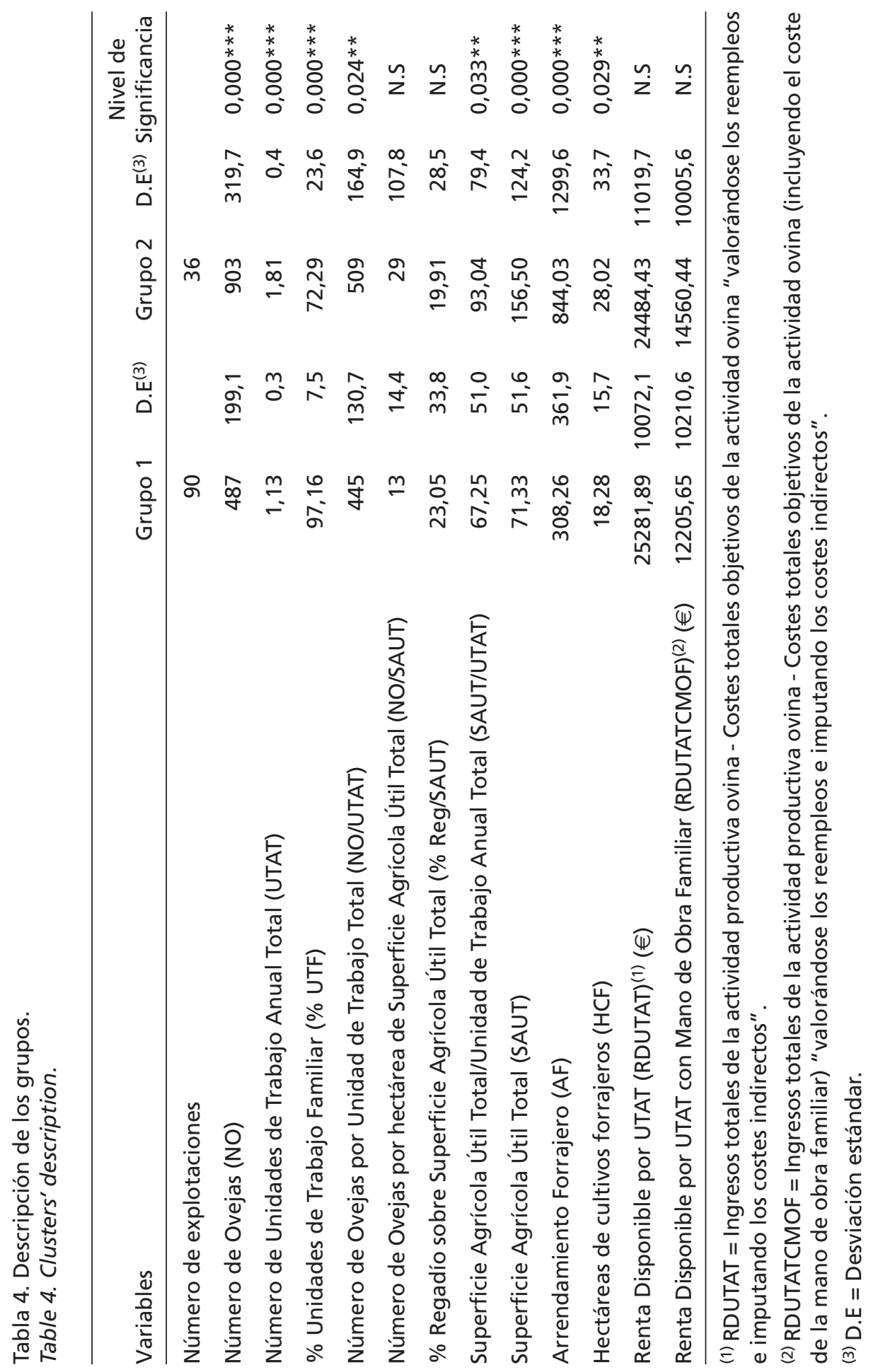


de obra familiar y son menos intensivas en el uso del trabajo al presentar menor número de ovejas y menor superficie agrícola útil por unidad de trabajo total. También son explotaciones con menor arrendamiento forrajero y menores hectáreas de cultivos forrajeros.

El grupo 2, por lo contrario es más reducido ( $28,6 \%$ de las explotaciones) y a él pertenecen explotaciones más grandes en cuanto al tamaño del rebaño, a la superficie agrícola total y al número de unidades de trabajo total. También realizan mayor arrendamiento forrajero, con mayores hectáreas de cultivos forrajeros y son más intensivas en la utilización de la mano de obra con mayor número de ovejas y mayor superficie agrícola total por unidad de mano de obra.

\section{Regresión lineal múltiple}

Como se mencionó en el apartado de metodología, las variables estructurales utilizadas en el análisis factorial se utilizaron como variables predictoras y la variable renta disponible por unidad de trabajo total sin tener en cuenta la remuneración de la mano de obra familiar (RDUTAT) y teniéndola (RDUTATCMOF) se eligió como variable respuesta o variable dependiente. Se analizaron las relaciones en cada uno de los grupos obtenidos, y también se efectuó el análisis de regresión sobre la totalidad de la muestra.

Los resultados de las seis regresiones se muestran en las tablas 5, 6 y 7. Como se puede observar, todos los estadísticos de evaluación de los modelos referidos a las seis regresiones realizadas son significativos. Los resultados se presentan a continuación.

Para la totalidad de la muestra (tabla 5), el modelo de regresión 1 (RDUTAT), en el que no se ha tenido en cuenta la remuneración de la mano de obra familiar en el cálculo de la renta disponible por unidad de trabajo, los resultados indican que la intensificación del trabajo con respecto al tamaño del rebaño
(NO/UTAT) y el porcentaje de la mano de obra familiar (\% UTF) tienen un impacto positivo en los resultados alcanzados. En el modelo de regresión 2 (RDUTATCMOF) donde a diferencia del anterior se tiene en cuenta el coste de oportunidad de la mano de obra familiar, sólo la intensificación del trabajo con respecto al tamaño del rebaño (NO/UTAT) tiene un impacto positivo en la renta disponible por unidad total de mano de obra. En ambos modelos, la carga ganadera (NO/SAUT) influye negativamente en el resultado económico. Una posible explicación de este resultado reside en que las explotaciones con mayor carga ganadera tienen menos hectáreas para responder a las necesidades del rebaño; por lo que necesitan comprar más alimentos para ser suministrados en pesebre o recurrir al arrendamiento de pastos. Chabosseau et al. (2000) encontraron que la carga ganadera afecta negativamente los resultados económicos de las explotaciones estudiadas debido al incremento en la compra de inputs como fertilizantes y concentrado.

En el grupo de explotaciones pequeñas y medianas, grupo 1, la intensificación de la mano de obra (NO/UTAT) (tabla 6), sigue siendo la variable que en mayor medida explica los mejores resultados económicos. También explican estos resultados el mayor número de hectáreas de SAUT y cuando en el cálculo no se tiene en cuenta la remuneración del coste de oportunidad de la mano de obra familiar, el menor número de hectáreas de cultivos forrajeros. Por tanto, en las explotaciones de menor tamaño, donde predominan los sistemas mixtos que combinan ganado y cultivos comerciales (sobre todo cereales), la superficie puede ser un factor limitante. Una mayor superficie iría unida a una mayor autonomía alimenticia y unos mejores resultados económicos. La relación directa entre la mayor autonomía alimenticia de las explotaciones y la mejora de la rentabilidad ha sido puesta de manifiesto por diversos autores (Bernués et al., 2011; Ryschawy et al., 2013; Ripoll-Bosch et al., 2014). 


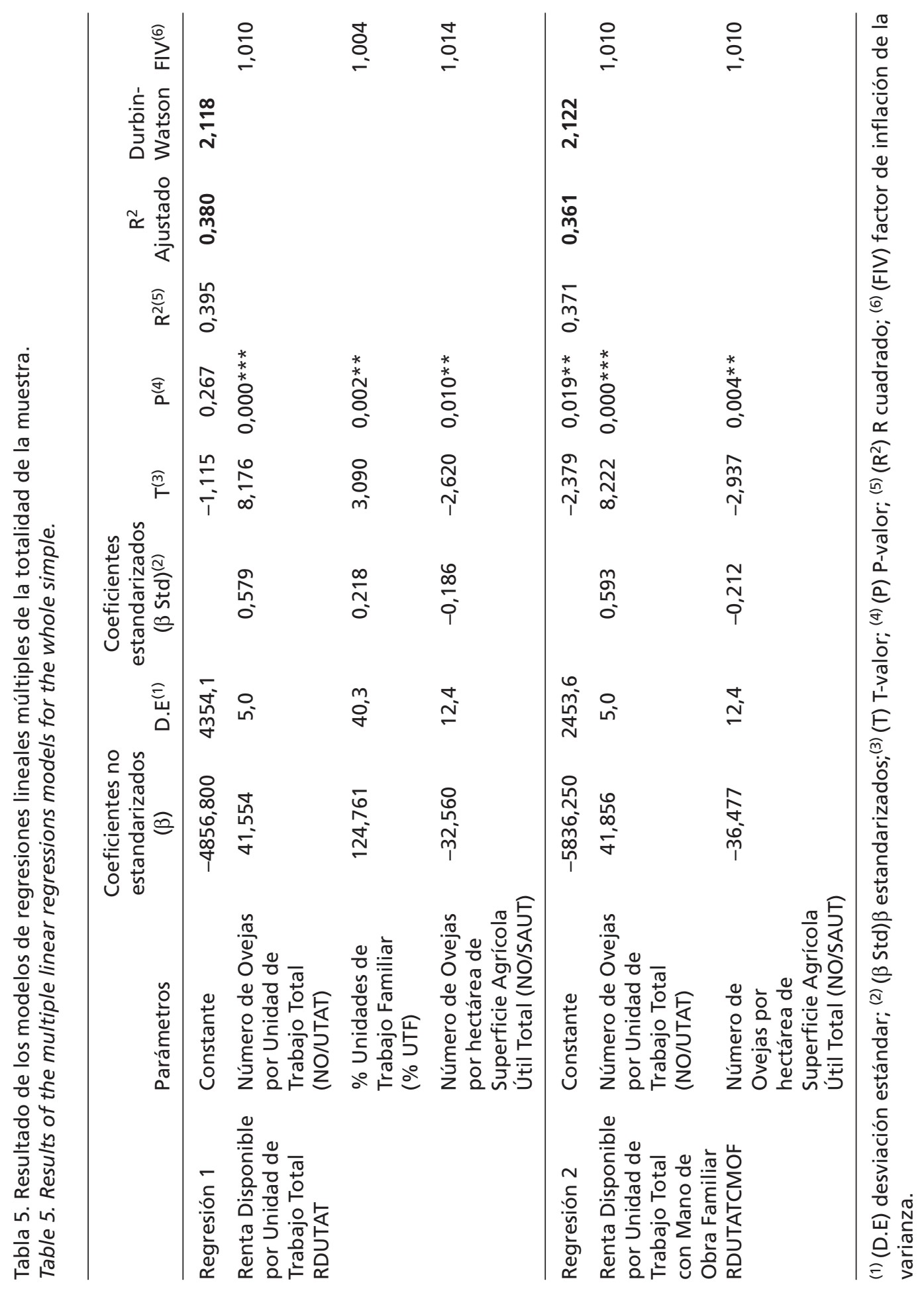




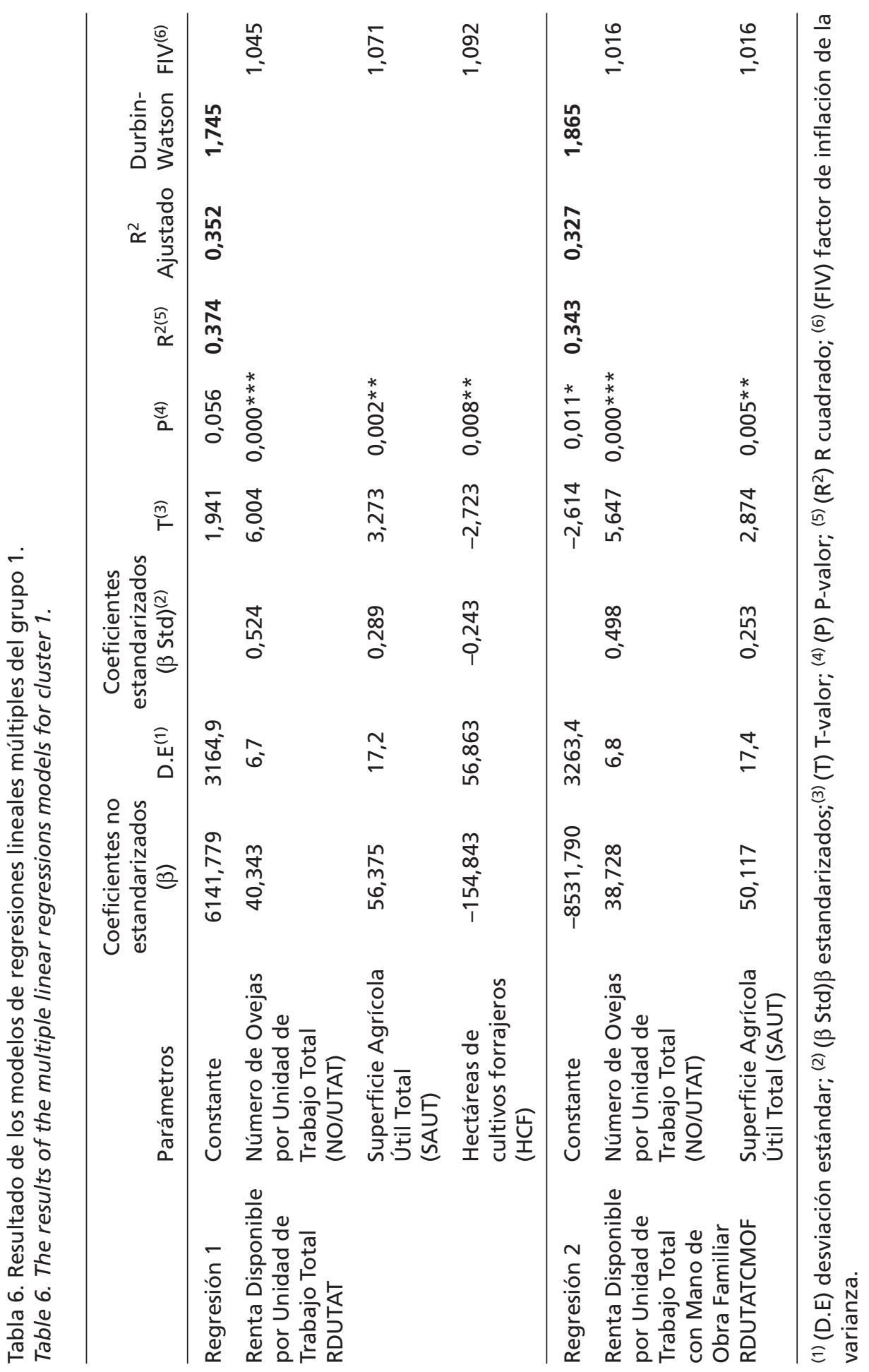




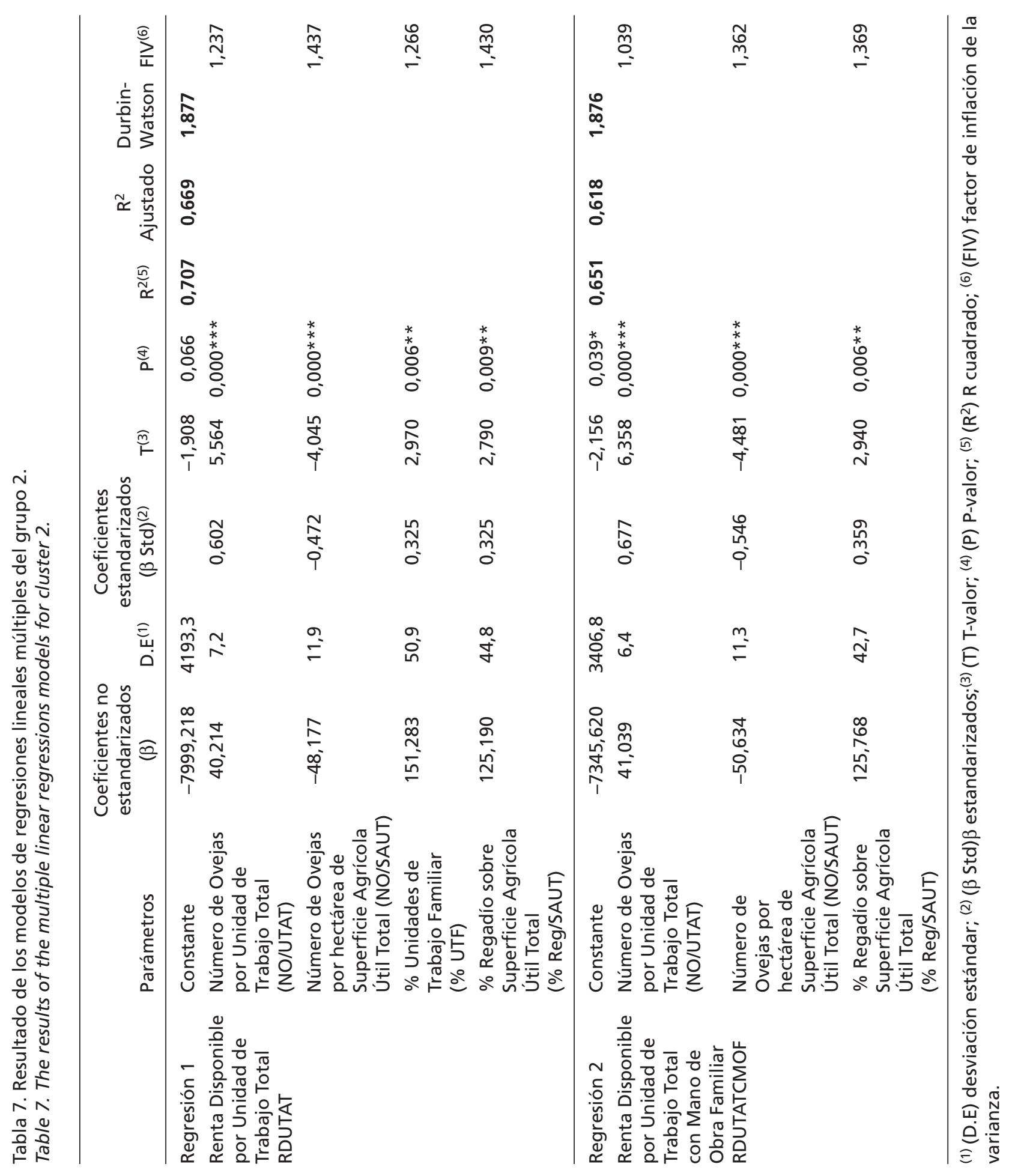


En el grupo de explotaciones más grandes (tabla 7), el grupo 2, los resultados coinciden con los del total de la muestra, siendo la intensificación de la mano de obra (NO/UTAT) una variable explicativa de unos mejores resultados, mientras que el mayor número de ovejas por hectárea de SAUT, de nuevo vuelve a aparecer con signo negativo. Como diferencia con respecto a los resultados de la totalidad de la muestra podemos destacar que en este grupo de explotaciones grandes, la mayor proporción de regadío (\% Reg/SAUT), se asocia también con unos mejores resultados económicos al permitir mejorar su autonomía alimenticia.

Los resultados del presente trabajo indican que la intensificación de la mano de obra está positivamente correlacionada con la renta disponible por unidad total de trabajo en todos los modelos analizados, por lo que se convierte en un instrumento importante para mejorar los resultados económicos por unidad de trabajo. De acuerdo con Pardos y Fantova (2007) las explotaciones con mejores resultados económicos son explotaciones con más ovejas por unidad de trabajo. Sin embargo, en opinión de algunos autores (Benoit y Laignel, 2011), la intensificación del trabajo no siempre lleva consigo más renta disponible por unidad total de trabajo, ya que no es fácil en esas circunstancias mantener el rendimiento zootécnico alto, por lo que es importante tener en cuenta el aspecto técnico cuando las explotaciones optan por la intensificación de la mano de obra. Además, el efecto positivo de la intensificación de la mano de obra sobre la renta disponible por unidad total del trabajo podría explicarse indirectamente por el tamaño del rebaño y las subvenciones de la PAC. De acuerdo con Lorent et al. (2009), las subvenciones acopladas estimulaban el crecimiento del tamaño del rebaño, por lo tanto, el número de ovejas por unidad total de mano de obra.
Con respecto a las unidades de trabajo familiar, éstas tienen un impacto positivo en la renta disponible por unidad de trabajo total solo cuando su coste no está incluido en la estimación del resultado económico. Este resultado se ha obtenido cuando se hizo el análisis de regresión sobre la totalidad de la muestra (tabla 5) y sobre el grupo 2 formado por las explotaciones más grandes (tabla 7). Este resultado es totalmente lógico e indica que solamente cuando no se retribuye la mano de obra familiar un mayor porcentaje de su utilización conlleva una mayor renta disponible. Coincide con lo señalado por otros autores Aggelopoulos et al. (2009) que han indicado la necesidad de reducir los costes de la mano de obra para la obtención de unos mejores resultados económicos. Esto no se observa en las explotaciones de menor tamaño donde la mayoría de la mano de obra es familiar. Por el contario estas explotaciones encuentran su limitación en las hectáreas de superficie útil disponible, por lo que una mayor disponibilidad explica unos mejores resultados.

Se puede señalar que según los resultados de la regresión del grupo 1, la variable SAUT está correlacionada positivamente con la RDUTAT con y sin tener en cuenta la mano de obra familiar. Mientras, la variable HCF tiene un poder negativo sobre la RDUTAT únicamente, cuando no se tiene en cuenta la mano de obra familiar, al incrementarse la importancia relativa del coste de alimentación en los costes totales. Como ya se ha indicado, este hecho podría tener su explicación en que estos sistemas, que suelen ser mixtos, intentan conseguir la autonomía forrajera sobre el aprovechamiento de los pastos naturales más que de los cultivos forrajeros, al orientar su SAU a la producción de cereales, tanto para la venta como para autoconsumo propio. 


\section{Conclusiones}

La tipificación estructural realizada ha permitido la clasificación de las explotaciones en dos grupos distintos que se diferencian fundamentalmente por su tamaño. Las explotaciones más pequeñas utilizan mayor proporción de trabajo familiar y realizan menores arrendamientos de superficie forrajera, mientras que las de mayor tamaño, son más intensivas en el uso del factor trabajo. Sin embargo, estas diferencias estructurales no se traducen en unos resultados económicos distintos, no siendo por tanto el tamaño un determinante de su rentabilidad.

Al relacionar los resultados económicos con las variables estructurales se observa que éstos guardan relación con una mayor intensificación del factor trabajo con respecto al tamaño del rebaño. Un mayor porcentaje de utilización de mano de obra familiar contribuye a unos mejores resultados cuando ésta no se remunera tanto en el grupo de explotaciones grandes como para el conjunto de la muestra. En las explotaciones pequeñas los mejores resultados económicos se asocian a una mayor disponibilidad de superficie, al tratarse probablemente de un factor limitante. Por el contrario, una alta carga ganadera por hectárea de superficie agrícola útil se relaciona con peores resultados económicos. Si bien la intensificación del trabajo se ha relacionado en muchos trabajos con unos mejores resultados económicos, sería preciso profundizar en los aspectos técnicos relacionados con la utilización de recursos y el manejo de las explotaciones para una mejor comprensión de la repercusión negativa que una mayor carga ganadera tiene sobre los resultados.

\section{Material complementario}

El material complementario de este artículo se puede consultar en la URL https://doi.org/ 10.12706/itea.2020.023

\section{Referencias bibliográficas}

Aggelopoulos S, Soutsas K, Pavloudi A, Sinapis E, Petkou D (2009). Suggestions for reformation in sheep farms based on production cost. Journal of Food, Agriculture and Environment 7(3-4): 561-566.

Andersen E, Elbersen B, Godeschalk F, Verhoog D (2007). Farm management indicators and farm typologies as a basis for assessments in a changing policy environment. Journal of Environmental Management 82: 353-362. https://doi. org/10.1016/j.jenvman.2006.04.021

Benoit M, Laignel G (2011). Analyse sur le long terme de systèmes d'élevage ovins allaitants en France. Quelles trajectoires et quels facteurs de réussite économique? INRA Productions Animales 24(3): 211-220. https://doi.org/10.20870/productions-animales.2011.24.3.3256

Bernués A, Ruiz R, Olaizola A, Villalba D, Casasús I (2011). Sustainability of pasture-based livestock farming systems in the European Mediterranean context: synergies and trade-offs. Livestock Science 139: 44-57. https://doi.org/ 10.1016/j.livsci.2011.03.018

Bernués A, Olaizola A (2012). La ganadería en los pirineos: Evolución, condicionantes y oportunidades. En: Los Pirineos Geografía, Turismo, Agricultura, Cooperación transfronteriza y Derecho (Ed. Lasagabaster I), pp. 29-67. Universidad del País Vasco.

Bernués $A$, Rodríguez-Ortega T, Martín-Collado $D$, Casasús I, Olaizola AM (2018). Evaluación de sostenibilidad en ovino de carne. XLIII Congreso Nacional y XIX Internacional de la Sociedad Española de Ovinotecnia y Caprinotecnia (SEOC), 19-21 de septiembre, Zaragoza, España, pp. 29-43.

Chabosseau JM, Laignel G, Souille C, Bergeron C, Lamoureuex J, Staub A (2000). Baisser le chargement par agrandissement des surfaces améliore les résultats économiques ovins en Montmorillonnais. Fourrages 161: 23-38.

Chertouh T (2005). Análisis de explotaciones ovinas aragonesas y viabilidad económica de la adopción de innovaciones en el sistema de alimentación. Tesis doctoral. Universidad de Zaragoza, España. 256 pp. 
Dubeuf JP, AW-Hassan A, Chentouf M, Mena Y, Pacheco F, Boutonnet JP (2016). The Mediterranean sheep and goat sectors between constants and changes over the last decade. Future challenges and prospects. Options Méditerranéennes: Série $A$. Séminaires Méditerranéens 115: 43-52.

EUROSTAT (2019). Sheep and goat market situation. Data base. Disponible en: https://ec.europa.eu/eurostat/web/agriculture/data/database (Consultado: 15 de diciembre 2019).

Gaspar P, Escribano M, Mesías FJ, Rodríguez de Ledesma A, Pulido $F$ (2008). Sheep farms in the Spanish rangelands (dehesas): Typologies according to livestock management and economic indicators. Small Ruminant Research 74: 52-63. https://doi.org/10.1016/j.smallrumres. 2007.03.013

Gelasakis Al, Valergakis GE, Arsenos G, Banos G (2012). Description and typology of intensive Chios dairy sheep farms in Greece. Journal of Dairy Science 95: 3070-3079. http://dx.doi.org/ 10.3168/jds.2011-4975

Hair JF, Black WC, Babin BJ, Anderson RE (2014). Multivariate data analysis, $7^{\text {th }}$ Ed. Pearson New International Edition, EE.UU. 739 pp.

Hamrouni S (1993). Tipificación estructural, técnica y económica y nivel de intensificación de las explotaciones ovinas del valle medio del Ebro en el marco y dinámica de las agriculturas desarrolladas. Tesis doctoral. Universidad de Zaragoza, España. 422 pp.

López-i-Gelats F, Milán MJ, Bartolomé J (2011). Is farming enough in mountain areas? Farm diversification in the Pyrenees. Land Use Policy 28: 783-791. https://doi.org/10.1016/j.landusepol.2011.01.005

Lorent $\mathrm{H}$, Sonnenschein R, Tsiourlis GM, Hostert $\mathrm{P}$, Lambin E (2009). Livestock subsidies and rangeland degradation in central Crete. Ecology and Society 14(2): 41. https://www.jstor.org/stable/26268320

MAGRAMA (2016). Foro Europeo Ovino De Carne 2015-2016. Aportaciones Ministerio De Agricultura, Alimentación Y Medio Ambiente, Dirección General De Producciones $Y$ Mercados Agrarios, 5 pp.
MAPA (2019). El sector ovino y caprino en cifras: Principales indicadores económicos. Subdirección General de Productos Ganaderos, Dirección General de Producciones Y Mercados Agrarios, $71 \mathrm{pp}$.

Marín-Bernal AM, Navarro-Ríos MJ (2014). Análisis y diagnóstico de los sistemas de producción ovina en el sureste español. Archivos de Zootecnia 63(243): 519-529. https://doi.org/10.21 071/az.v63i243.531

Maza MT, Pardos L, Fantova E (2008). Analysis of the contribution of sales of Ternasco de Aragón PGI to the economic results of farms. Options Méditerranéennes: Série $A$. Séminaires Méditerranéens 78: 133-138.

Mena Y, Ruiz FA, Gutiérrez R, Vázquez M, Castel JM (2014). La función de la ganadería en el mantenimiento y el aprovechamiento de los montes mediterráneos. Ambienta 108: 52-67.

Olaizola A, Manrique E, Bernués A, Maza MT (1996). Incidence of programmes to discourage milk production on representative cattle farms in a less favoured area. Investigación Agraria, Economía 11: 355-376.

Olaizola A, Chertouh T, Manrique E (2008). Adoption of new feeding technology in Mediterranean sheep farming systems: Implications and economic evaluation. Small Ruminant Research 79: 137-145. https://doi.org/10.1016/j.smallrumres.2008.07.022

Olaizola A, Barrantes O, Manrique E, Reiné R, Ferrer C, Broca A, Mora JL, Usón A, Riaguas L, Oliván A, Fantova O, Nicholas PK (2014). Identificación de las principales características de los sistemas mixtos agro-ganaderos y posibles estrategias de innovación en el valle medio del Ebro. Pastos y PAC 2014-2020: 53ª reunión científica de la SEEP, 9-12 junio, Potes, Cantabria, España, pp. 507-514.

Pardos L, Fantova E (2007). Importancia del control de gestión técnico-económico en las ganaderías de ovino de carne: Influencia de los diferentes factores productivos en los resultados económicos. En: Producción de ovino de carne en medio semiárido (Ed. Folch J), pp.18-29, Grupo Consolidado de Investigación Aplicada sobre Producción de Ovino de Carne-Gobierno de Aragón, España. 
Pardos L, Maza MT, Fantova E, Sepúlveda W (2008). The diversity of sheep production systems in Aragón (Spain): Characterisation and typification of meat sheep farms. Spanish Journal of Agricultural Research 6(4): 497-507. https://doi.org/ 10.5424/sjar/2008064-344

Pérez C (2005). Métodos estadísticos avanzados con SPSS. Ediciones Paraninfo Madrid, España. $792 \mathrm{pp}$.

Pineda Jaimes NB, Bosque Sendra J, Gómez Delgado M, Franco Plata R (2011). Análisis de los factores inductores de los cambios ocurridos en la superficie forestal del estado de México en el periodo 1993-2000. Boletín de la asociación de Geógrafos Españoles 56: 9-34.

Ripoll-Bosch R, Joy M, Bernués A (2014). Role of selfsufficiency, productivity and diversification on the economic sustainability of farming systems with autochthonous sheep breeds in less favoured areas in Southern Europe. Animal 8: 12291237. https://doi.org/10.1017/S1751731113000529

Rodríguez-Ortega T, Martín-Collado D, Casasús I, Olaizola A, Bernués A (2018a). Análisis multi-actor de la sostenibilidad y viabilidad del sector ovino de carne en Aragón. 57ª Reunión Científica de la Sociedad Española de Pastos: Pastoralismo y vias pecuarias, Conectando tradición e innovación, 25-29 junio, Teruel, España, pp. 339-347.

Rodríguez-Ortega T, Olaizola A, Bernués A (2018b). A novel management-based system of payments for ecosystem services for targeted agri-environmental policy. Ecosystem Servives 34: 74-84. https://doi.org/10.1016/j.ecoser.2018.09.007

Ruiz-Mirazo J, Robles AB, González-Rebollar JL (2011). Two-years evaluation of fuelbreaks grazed by livestock in the wildfire prevention pro- gram in Andalusia (Spain). Agriculture, Ecosystems and Environment 141: 13-22. https://doi. org/10.1016/j.agee.2011.02.002

Ryschawy J, Choisis N, Choisis JP, Gibon A (2013). Paths to last in mixed crop-livestock farming: lessons from an assessment of farm trajectories of change. Animal 7: 673-681. https://doi.org /10.1017/S1751731112002091

Sakar E, Keskin, S, Unver H (2011). Using of factor analysis scores in multiple linear regression model for prediction of kernel weight in Ankara walnuts. The Journal of Animal \& Plant Sciences 21(2): 182-185.

Tirel JC (1983). Le débat sur le productivisme. Économie Rurale 155: 23-30.

Toro-Mujica MP, Aguilar C, Vera R, Barba C, Rivas J, García-Martínez A (2015). Changes in the pastoral sheep systems of semi-arid Mediterranean areas: association with common agricultural policy reform and implications for sustainability. Spanish Journal of Agricultural Research 13(2): 1-11. https://doi.org/10.5424/sjar/2015132-6984

Valerio Cabrera D, García Martínez A, Acero de la Cruz R, Castaldo A, Perea JM, Martos Peinado J (2004). Metodología para la caracterización y tipificación de sistemas ganaderos. Documento de Trabajo, Producción Animal y Gestión 1: 1-9

Vázquez González I, Sineiro García F, García Arias Al (2014). Trayectorias de crecimiento de las explotaciones agrarias en la Cornisa Cantábrica. Economía Agraria y Recursos Naturales (2): 4980. https://doi.org/10.7201/earn.2014.02.03

(Aceptado para publicación el 13 de julio de 2020) 